\title{
Products Refurbishment and its Logistics in Environment Conscious Product Lifecycle Management
}

\author{
Kenji YURA ${ }^{1}$ \\ ${ }^{1}$ Department of Systems Engineering, University of Electro-Communications, Japan, yura@se.uec.ac.jp
}

\begin{abstract}
:
Products refurbishment and the logistic systems are discussed toward environment conscious product lifecycle management. Three alternative systems are enumerated and their environmental impacts are compared. The first system implements the refurbishment at the products users, the second does it at the dealers, and the third does it at the manufacturer. Depending on the place performing it, the necessary logistic systems are varied. Further, a performance level of the refurbishment depends not only on the place but also on the products structure. Then, the environmental impacts from the systems are formulated and dominance relations among the systems are clarified.
\end{abstract}

Keywords: Refurbishment, Logistics, Environmental impacts, PLM, Modular design

\section{Introduction}

One of our issues encountered in these days is to keep and improve the modern life while the sound environment is sustained. For tackling with the issue, various approaches have been proposed and developed to decrease the environmental impacts (e.g. generation of greenhouse gas) over the products lifecycle. One of them is to develop recycling-oriented manufacturing systems, where used-products are disassembled and the available parts or material are reused or recycled (e.g.[1]). However, the system still consumes considerable energy and affects the environment from the forward and backward logistics of products.

The flow of products must be reduced to realize more environmental-conscious manufacturing, however, there is an inevitable fact that most products become obsolete or failed, and the users need to buy new ones. Recently, progress of ICT enables to construct PLM (Product Lifecycle Management: e.g. [2]) system and it could allow users to manage the obsolete products in an environment friendly manner. Then, three systems to refurbish the obsolete products are proposed to reduce the products flow in this article. The first system implements the refurbishment at the user, the second does it at the dealer, and the third does it at the manufacturer.

In the refurbishment, the obsolete product is firstly disassembled, next the obsolete components of the product are replaced with new ones, and then the product is reassembled. In the disassembly planning, Disassembly Levelling (DL) and Disassembly Process Planning (DPP) need to be determined $[3,4]$. The former relates to a disassembly depth, which shows how far the product should be disassembled, e.g. the product is disassembled to components, sub-components, or parts.

Modular design [5] is closely related to the disassembly levelling. Modular design simplifies disassembly operation and components replacement for a product. Of course, it affects the environmental impact from the module replaced at the refurbishment.
In the three types of refurbishment systems, technological abilities of the sites (the user, the dealer, and the manufacturer) are different, and then the module replaced in the refurbishment operation is also different from site to site. Therefore, environmental impacts from the refurbishment and logistics of replaced module depend on where the operation is implemented and what the module is replaced at the site. Taking the above points into consideration, the environmental performances of the three refurbishment systems are formulated and the performances are compared in the following sections.

\section{Models of Refurbishment Systems}

\subsection{Alternative Refurbishment Systems}

In recycling-oriented manufacturing systems, products flow over the life cycle is one-directional such as shown in Figure 1. A product is produced at a manufacturer, and it is transported to a user through a dealer. The user utilizes and maintains the product with supports of the dealer and/or the manufacturer. When the product is obsolete or failed and the user decides to dump the product, the used product is shipped to a recycler, and there it is disassembled into components. After testing and cleaning the dismantled units, the available units are transported to the manufacturer as the reusable ones. The residuals are processed and they turn out raw material.

Such the recycling-oriented manufacturing system is effective to reduce the environmental impacts not only from producing virgin material but also from dumping used-product. However, considerable energy is still consumed both in the delivery of new products and in the collection of used-products.

Failures and obsolescence are inevitable matters for most products. However, it is unnecessary to recycle the whole product. Replacing the obsolete components with new ones, the old product can be renewed and the renewed one is used at the user. It prolongs the product lifecycle as shown in Figure 2. Further, the amount of materials to be recycled can be reduced. Such the activity 
is called refurbishment operation of products.

In this article, three alternative systems implementing the refurbishment operations are proposed to reduce the environmental impacts. Suppose the product structure is tree type as shown in Figure 3, where the product has a hierarchical structure and it consists of several modules with level 1, each of the modules is composed of modules with level 2, the module with level 2 is assembled from modules with level 3, and so on. Then, the alternative systems are described as follows.

(System A): The refurbishment operation is performed at a user. A new module (e.g. Module 2 in Figure 3) is supplied to the user, the module is replaced with the old module, and the old module is sent to the recycler (see Figure 4). In order to enable this system, the product should be designed so that the module can be easily replaced, and necessary tools and the operations manual must be available for the user.

(System B) : The refurbishment operation is performed at a dealer. The product needs to be transported from the user to the dealer. A new module (e.g. Module 22) is supplied to the dealer, the module is replaced with the old module, and the old module is sent to the recycler (see Figure 5). In order to enable this method, necessary facilities/tools and the operations manual should be available for the dealer. When the operation completes, the product is sent to the user. This system requires the transportation of the products between the user and the dealer, which is unnecessary in system $A$.

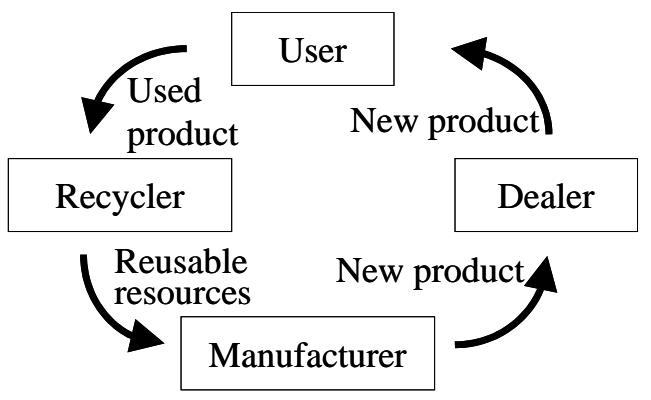

Figure 1: Products flow in recycling-oriented manufacturing systems

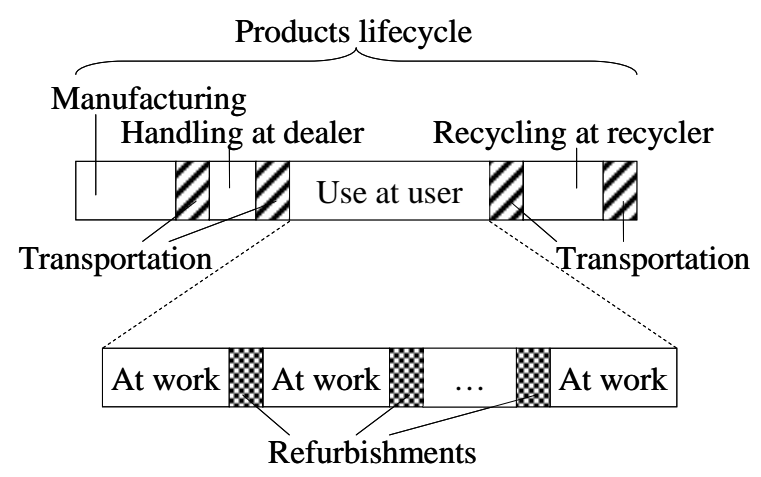

Figure 2: Product lifecycle and refurbishments in products use
(System $C$ ) : The refurbishment operation is performed at a manufacturer. The product needs to be transported to the manufacturer. A new module (e.g. Module 222) is replaced with the old module, and the old module is sent to the recycler (see Figure 6). When the operation completes, the product is sent back to the user. This system requires the transportation of the products between the user and the manufacturer, which is unnecessary in systems $A$ and $\mathrm{B}$.

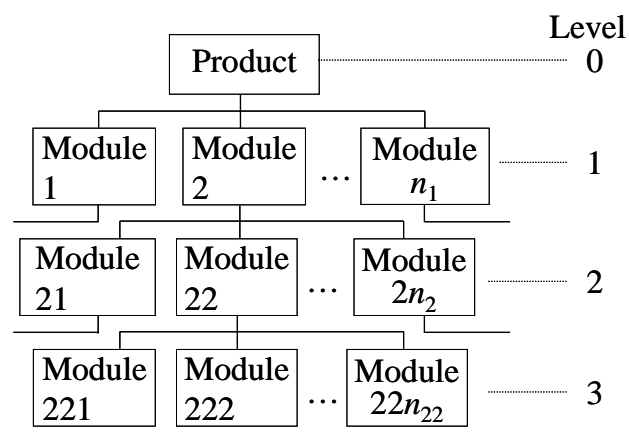

Figure 3: Product structure and modules

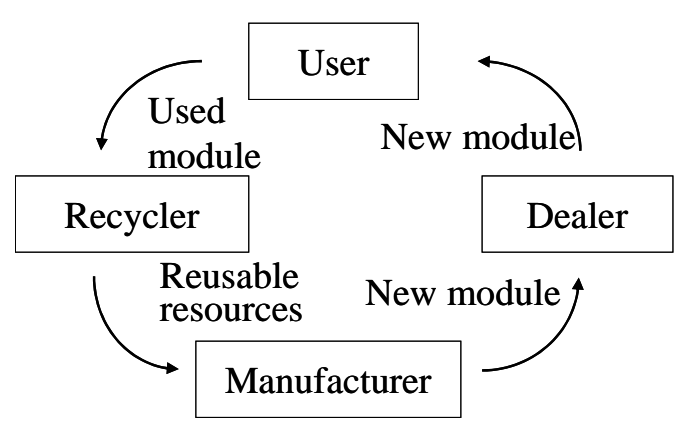

Figure 4: Modules flow in refurbishment system $A$

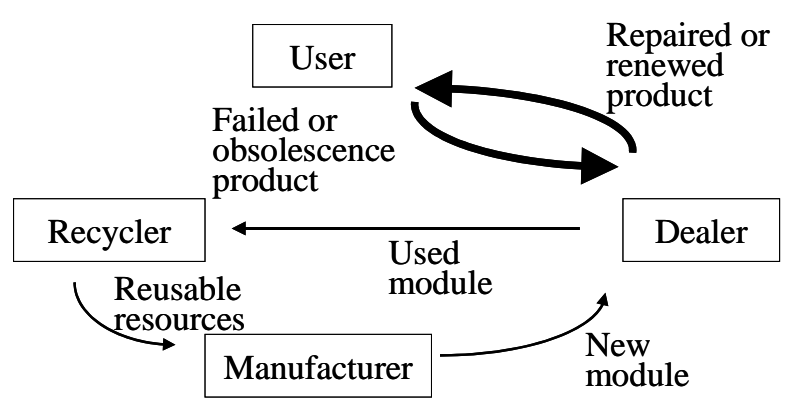

Figure 5: Modules flow in refurbishment system $B$

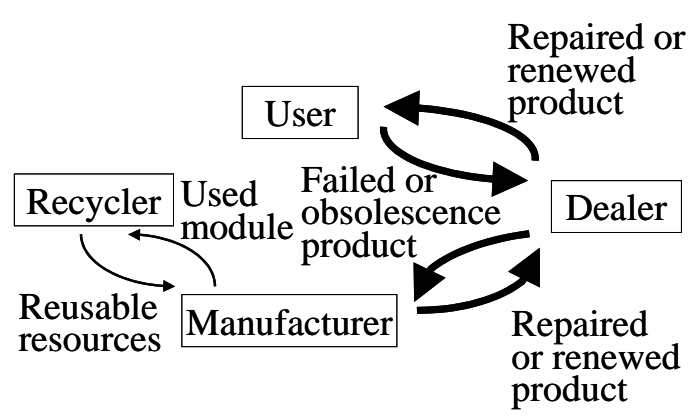

Figure 6: Modules flow in refurbishment system $C$ 


\subsection{Environmental Impact over Product Lifecycle}

The following symbols are used to formulate the total environmental impacts over the product life cycle for three refurbishment systems $A, B$, and $C$, and the recycling-oriented manufacturing system without refurbishment, which is denoted as system null.

$E(i)$ : total environmental impact of a product by refurbishment system $i$ ( $i=A, B, C$ or null) .

$M$ : environmental impact from making a product at the manufacturer.

$T_{J K}$ : environmental impact from transporting a product between cite $J$ and cite $K(J, K=M$ (manufacturer), $D$ (dealer), $U$ (user), or $R$ (recycler)).

$U(i)$ : environmental impact from the product usage at the user in system $i$.

$R$ : environmental impact from recycling a product at the recycler.

$\varepsilon(i)$ : total environmental impact per period in system $i$.

$L(i)$ : length of the products life cycle in system $i$.

The total environmental impact, $E(i)$, is given by the sum of the impacts in each stage of the product life cycle, such as manufacturing, handling at the dealer, usage, recycling, and transportations (refer to Figure 2).

$$
E(i)=M+T_{M D}+T_{D U}+U(i)+T_{U R}+R+T_{R M}
$$

The total environmental impact per period by system $i$, $\varepsilon(i)$, is given by the following equation using the length of the product lifecycle $L(i)$.

$$
\varepsilon(i)=E(i) / L(i)
$$

\subsection{Environmental Impact from Product-usage Stage}

The environmental impact from the product-usage stage in the lifecycle, $U(i)$, is different from system to system. The following symbols are used in order to formulate $U(i)$.

$N(i)$ : number of refurbishment operations in the product lifecycle of system $i$.

$v(i)$ : environmental impact per period from using the product in system $i$.

$m(i)$ : environmental impact from making the module, which is replaced at the refurbishment operation of system $i$. The value of $m(i)$ is not greater than $M$.

$t_{J K}(i)$ : environmental impact from transporting the module between cite $J$ and cite $K$ in system $i \quad(J, K=$ $M$ (manufacturer), $D$ (dealer), $U$ (user), or $R$ (recycler)). The value of $t_{J K}(i)$ is not greater than $T_{J K}$. $f(i)$ : environmental impact from the refurbishment operation in system $i$.

$r(i)$ : environmental impact from recycling the module at the recycler in system $i$, where $r(i) \leq R$.

The impact $U(i)$ is the sum of two impacts, where the one occurs from using the product and the other from refurbishing it. The refurbishment related activities are composed of manufacturing, recycling and transportation of the module, transportation of the product to the refurbishment cite, and the refurbishment operation itself.
The transportation routes of the module and the product are given for systems A, B, and C in Figures 4-6, respectively. Then, $U(i)$ is formulated as follows.

$$
\begin{aligned}
& U(A)=L(A) v(A)+N(A)\left[m(A)+t_{M D}(A)+t_{D U}(A)\right. \\
&\left.+f(A)+t_{U R}(A)+r(A)+t_{R M}(A)\right] \\
& U(B)=L(B) v(B)+N(B)\left[m(B)+t_{M D}(B)+T_{U D}\right. \\
&\left.+f(B)+T_{D U}+t_{D R}(B)+r(B)+t_{R M}(B)\right] \\
& U(C)=L(C) v(C)+N(C)\left[m(C)+T_{U D}+T_{D M}+f(C)\right. \\
&\left.+T_{M D}+T_{D U}+t_{M R}(C)+r(C)+t_{R M}(C)\right] \\
& U(\text { null })=L(\text { null }) v(\text { null })
\end{aligned}
$$

\section{Analysis}

\subsection{Effect of Refurbishment Systems}

The effect of refurbishment systems on reducing the environmental impacts is evaluated in this section.

The environmental impact per period, $\varepsilon(i)$, is obtained by substituting Eq.(1) into Eq.(2) as follows.

$$
\begin{array}{r}
\varepsilon(i)=\left[M+T_{M D}+T_{D U}+U(i)+T_{U R}+R+T_{R M}\right] / L(i) \\
(i=A, B, C, \text { null })
\end{array}
$$

Then, the relation between $\varepsilon(i)(i=A, B, C)$ and $\varepsilon($ null $)$ is shown as follows.

$$
\varepsilon(i)=[\varepsilon(\text { null }) L(\text { null })-U(\text { null })+U(i)] / L(i)
$$

Using Eq.(8), the difference between $\varepsilon$ (null) and $\varepsilon(i)$, which is denoted by $\Delta \varepsilon_{0 i}(i=A, B, C)$, is shown as follows.

$$
\Delta \varepsilon_{0 i}=\{\varepsilon(\text { null })[L(i)-L(\text { null })]-[U(i)-U(\text { null })]\} / L(i)
$$

Therefore, if $\varepsilon($ null $)>[U(i)-U($ null $)] /[L(i)-L($ null $)]$, system $i$ is superior to the system without refurbishment.

Let $\xi$ denote the value of $M+T_{M D}+T_{D U}+T_{U R}+R+$ $T_{R M}$, i.e. $\xi=E(i)-U(i)$, and $\eta(i, 0)(>1)$ denote the ratio of $L(i) / L($ null $)$. From the definition, $\Delta \varepsilon_{0 i}$ is expressed as follows.

$$
\begin{aligned}
\Delta \varepsilon_{0 i} & =[E(\text { null }) / L(\text { null })]-[E(i) / L(i)] \\
& =\{\xi+U(\text { null })-[\xi+U(i)] / \eta(i, 0)\} / L(\text { null })
\end{aligned}
$$

Then, if the following relation holds, system $i$ is superior to the system without refurbishment.

$$
\xi>[U(i)-\eta(i, 0) U(\text { null })] /[\eta(i, 0)-1](i=A, B, C)
$$

In case that $v(i)=v($ null $)$ and $L(i)=[N(i)+1] L($ null $)$, the condition that $\Delta \varepsilon_{0 i}$ becomes positive is, $\xi>m(A)+$ $t_{M D}(A)+t_{D U}(A)+f(A)+t_{U R}(A)+r(A)+t_{R M}(A)$ for $i=A, \xi>$ $m(B)+t_{M D}(B)+T_{U D}+f(B)+T_{D U}+t_{D R}(B)+r(B)+t_{R M}(B)$ for $i=B$, and $\xi>m(C)+T_{U D}+T_{D M}+f(C)+T_{M D}+T_{D U}+$ $t_{M R}(C)+r(C)+t_{R M}(C)$ for $i=C$. Then, if $M+R>m(A)+$ $f(A)+r(A)$, system $A$ is superior to system null. However, systems $B$ and $C$ are not always superior to system null, even if $M+R>m(i)+f(i)+r(i)$ for $i=B$ and $i=C$.

\subsection{Comparison of Refurbishment Systems}

The environmental performances of the refurbishment systems are compared. Let $\Delta \varepsilon i j$ denote the difference of 
$\varepsilon(i)$ and $\varepsilon(j)$, and $\eta(i, j)$ be the ratio of $L(i) / L(j)$. Then,

$$
\begin{aligned}
& \Delta \varepsilon i j=[1 / L(i)-1 / L(j)] \xi+U(i) / L(i)-U(j) / L(j) \\
&=\{[1-\eta(i, j)] \xi+[U(i)-\eta(i, j) U(j)]\} / L(i) \\
&(i, j=A, B, C)
\end{aligned}
$$

When $L(i)$ equals $L(j)$, i.e. $\eta(i, j)=1$, system $i$ is superior to system $j$ for $U(i)<U(j)$ and vice verse. When $L(i)$ is greater than $L(j)$, i.e. $\eta(i, j)>1$, system $i$ is superior to system $j$ for $[\eta(i, j)-1] \xi<U(i)-\eta(i, j) U(j)$ and vice verse. On the other hand, when $L(i)$ is less than $L(j)$, i.e. $\eta(i, j)<1$, systems $i$ is superior to system $j$ for $[1-\eta(i, j)] \xi$ $<U(i)-\eta(i, j) U(j)$ and vice verse.

In case that $L(A)=L(B)=L(C), N(A)=N(B)=N(C)$, and $v(A)=v(B)=v(C)$, the superiority of systems are obtained as follows. Let $\delta_{i j}(i, j=A, B, C)$ denote the difference between the environmental impacts from handling the modules in systems $i$ and $j$ as shown Eq.(13), and $\Delta U_{i j}$ be the difference $U(i)-U(j)$. Then, from Eqns.(3) to (5), $\Delta U_{i j}$ is obtained as Eqns.(14) to (16) .

$$
\begin{aligned}
\delta_{i j}=[ & m(i)+f(i)+r(i)]-[m(j)+f(j)+r(j)] \\
\Delta U_{A B}= & \delta A B+\left[t_{M D}(A)-t_{M D}(B)\right]+\left[t_{R M}(A)-t_{R M}(B)\right] \\
& -\left[T_{D U}-t_{D U}(A)\right]-\left[T_{U D}+t_{D R}(B)-t_{U R}(A)\right] \\
\Delta U_{A C}= & \delta A C-\left[T_{M D}-t_{M D}(A)\right]-\left[T_{D U}-t_{D U}(A)\right] \\
& -\left[T_{U D}+T_{D M}+t_{M R}(C)-t_{U R}(A)\right] \\
\Delta U_{B C}= & \delta B C-\left[T_{M D}-t_{M D}(B)\right]-\left[T_{D M}+t_{M R}(C)-t_{D R}(B)\right]
\end{aligned}
$$

As shown in Eqns.(14) to (16), the difference of environmental impacts consists of two factors. One is related to the handling of modules, and the other is related to transportations of a product and a module.

\section{Discussion}

Based on the relations obtained in section 3 , two cases are evaluated under the conditions that $L(A)=L(B)=L(C)$, $N(A)=N(B)=N(C)$, and $v(A)=v(B)=v(C)$.

(Case 1) Modules are same in systems $A, B$, and $C$ In the case, $\delta_{A B}=\delta_{A C}=\delta_{B C}=0$ and $\Delta U_{i j}$ is given by,

$$
\begin{aligned}
\Delta U_{A B}= & -\left[T_{D U}-t_{D U}\right]-\left[T_{U D}+t_{D R}-t_{U R}\right] \leq 0 \\
\Delta U_{A C}= & -\left[T_{M D}-t_{M D}\right]-\left[T_{D U}-t_{D U}\right]-\left[T_{U D}+T_{D M}\right. \\
& \left.+t_{M R}-t_{U R}\right] \leq 0 \\
\Delta U_{B C}= & -\left[T_{M D}-t_{M D}\right]-\left[T_{D M}+t_{M R}-t_{D R}\right] \leq 0
\end{aligned}
$$

From Eqns.(17) to (19), system $A$ is the best, system $B$ is the second best, and system $C$ is the last one.

(Case 2) Modules are not same in systems $A, B$, and $C$

In this case, there is no dominance relation. The best system depends not only on the modules but also on geographical locations among the manufacturer, dealer, user, and recycler. Then, it should be clarified how the geographical locations effects on the best selection from the alternatives. Suppose that the modules are given and the best system should be selected for each user by taking his/her location into account.

From Eq.(14), if the location of a user is far from the dealer and not so far from the recycler, system $A$ could be superior to system B for some values of $\delta A B$. From Eq.(16), if the location of a recycler is far from the manufacturer but not so far from the dealer, system $B$ could be superior to system $C$. In this case, the area of user's location can be decomposed as shown in Figure 7.

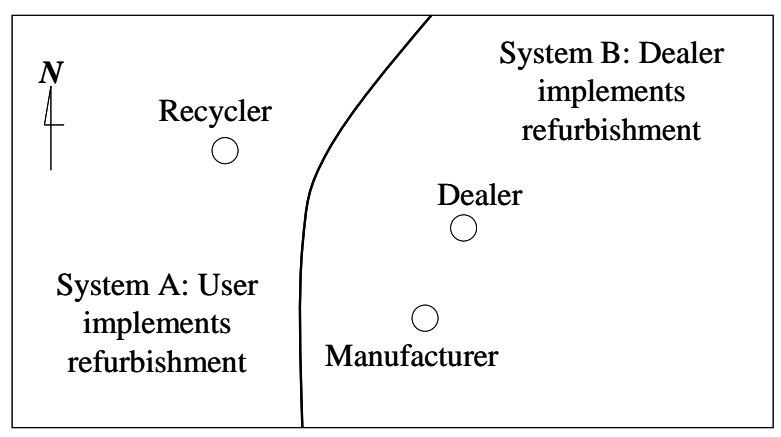

Figure 7: One of the possible decompositions of the area of users' places

\section{Conclusion}

This article shows the possibility that the refurbishment systems can reduce the current environmental impacts arising in the products lifecycle. Three types of the systems are enumerated and their environmental performances are formulated and compared. Then, some dominance relations are clarified for a special case. Further, it is shown that the best refurbishment system is different not only by the environmental impact of refurbishment but also the geographical allocation of the manufacturer, dealer, recycler and user. Then, it is important to teach each user where the obsolete product should be refurbished.

\section{Acknowledgement}

The author is grateful to the reviewers for their valuable comments. Also, the author wishes to thank the support by Grants-in-Aid for Scientific Research (B), No.17310095, from JSPS.

\section{References}

[1] Hoshino T., Yura K., and Hitomi K., 1995, Optimization Analysis for Recycle-Oriented Manufacturing Systems, IJPR, Vol.33, pp.2069-2078.

[2] Stark J., 2005, Product Lifecycle Management: $21^{\text {st }}$ Century Paradigm for Product Realisation (Springer).

[3] Lambert A.J.D., 2003, Disassembly Sequencing: A Survey, IJPR, Vol.41, pp.3721-3759.

[4] Gao M., Zhou M. C., and Caudill R. J., 2002, Integration of Disassembly Leveling and Bin Assignment for Demanufacturing Automation, IEEE Transactions on Robotics and Automation, Vol.18, pp.867-874.

[5] Huang C-C. and Kusiak A., 1998, Modularity in Design of Products and Systems, IEEE Transactions on Systems, Man and Cybernetics - Part A: Systems and Humans, Vol.28, pp.66-77. 